\title{
Six years of BeppoSAX Wide Field Cameras observations of nine galactic type I X-ray bursters
}

\author{
R. Cornelisse ${ }^{1,2}$, J. J. M. in 't Zand ${ }^{1}$, F. Verbunt ${ }^{2}$, E. Kuulkers ${ }^{3}$, J. Heise ${ }^{1}$, P. R. den Hartog ${ }^{2,1}$, M. Cocchi ${ }^{4}$, \\ L. Natalucci ${ }^{4}$, A. Bazzano ${ }^{4}$, and P. Ubertini ${ }^{4}$ \\ 1 SRON National Institute for Space Research, Sorbonnelaan 2, 3584 CA Utrecht, The Netherlands \\ 2 Astronomical Institute, PO Box 80000, 3508 TA Utrecht, The Netherlands \\ 3 ESA-ESTEC, SCI-SDG, Keplerlaan 1, 2201 AZ Noordwijk, The Netherlands \\ ${ }^{4}$ Istituto di Astrofisica Spaziale (CNR), Area Ricerca Roma Tor Vergata, Via del Fosso del Cavaliere, 00133 Roma, Italy
}

Received 21 February 2003 / Accepted 17 April 2003

\begin{abstract}
We present an overview of BeppoSAX Wide Field Cameras observations of the nine most frequent type I X-ray bursters in the Galactic center region. Six years of observations (from 1996 to 2002) have amounted to 7 Ms of Galactic center observations and the detection of 1823 bursts. The 3 most frequent bursters are GX 354-0 (423 bursts), KS 1731-260 (339) and GS 1826-24 (260). These numbers reflect an unique dataset. We show that all sources have the same global burst behavior as a function of luminosity. At the lowest luminosities $\left(L_{\mathrm{X}} \lesssim 2 \times 10^{37} \mathrm{erg} \mathrm{s}^{-1}\right)$ bursts occur quasi-periodically and the burst rate increases linearly with accretion rate (clear in e.g. GS 1826-24 and KS 1731-260). At $L_{\mathrm{pers}}=2 \times 10^{37} \mathrm{erg} \mathrm{s}^{-1}$ the burst rate drops by a factor of five. This corresponds to the transition from, on average, a hydrogen-rich to a pure helium environment in which the flashes originate that are responsible for the bursts. At higher luminosities the bursts recur irregularly; no bursts are observed at the highest luminosities. Our central finding is that most of the trends in bursting behavior are driven by the onset of stable hydrogen burning in the neutron star atmosphere. Furthermore, we notice three new observational fact which are difficult to explain with current burst theory: the presence of short pure-helium bursts at the lowest accretion regimes, the bimodal distribution of peak burst rates, and an accretion rate that is ten times higher than predicted at which the onset of stable hydrogen burning occurs. Finally, we note that our investigation is the first to signal quasi-periodic burst recurrence in KS 1731-260, and a clear proportionality between the frequency of the quasi-periodicity and the persistent flux in GS 1826-24 and KS 1731-260.
\end{abstract}

Key words. accretion, accretion disks - binaries: close - stars: neutron - X-rays: bursts

\section{Introduction}

Since the discovery of type I bursts by Grindlay \& Heise (1975) about 65 other X-ray bursters have been discovered (e.g., in 't Zand 2001). Most of these are concentrated toward the Galactic center, which illustrates their Galactic origin. X-ray bursts are characterized by a fast rise and an exponential decay with durations ranging from seconds to tens of minutes. Their spectrum can best be described by black body radiation with cooling during the decay of the burst. These type I X-ray bursts are due to unstable hydrogen/helium burning in a thin shell on a neutron star surface (see, e.g., the review by Lewin et al. 1993).

By far most of the X-ray bursts are emitted by sources, persistent or transient, with luminosities of $10^{36-37} \mathrm{erg} \mathrm{s}^{-1}$. Some sources at higher persistent luminosities also show X-ray bursts (e.g., Kuulkers et al. 2002), but such bursts are less common. At lower luminosities bursts have also been sporadically

Send offprint requests to: R. Cornelisse,

e-mail: R.Cornelisse@sron.nl observed (e.g., Gotthelf \& Kulkarni 1997; Cocchi et al. 2001a; Cornelisse et al. 2002).

Assuming that the amount of fuel burnt per burst is roughly the same, one expects that the burst rate increases linearly with accretion rate. However, for most X-ray bursters where it is possible to study this the opposite is observed (van Paradijs et al. 1988a), see e.g. GX 3+1 (den Hartog et al. 2002). Bildsten (2000) noted that the onset of a burst is governed by the local rather than the global accretion rate (see also Marshall 1982). If the area on the neutron star on which accretion takes place increases rapidly with the global accretion rate, the local accretion rate may actually drop, giving rise to a lower burst rate.

Observations of several sources (e.g., 4U 1705-44, Gottwald et al. 1989; EXO 0748-676, Gottwald et al. 1986), show different burst properties at different accretions rates. Fujimoto et al. (1981) predicted this behavior by showing that the composition of the unstable burning shell changes with accretion (see also Bildsten 1998 for a recent overview). Briefly, at the highest accretion rates $\left(\dot{M} \gtrsim 10^{-9} M_{\odot} \mathrm{yr}^{-1}\right)$ the helium 
ignites in an unstable fashion in a mixed $\mathrm{He} / \mathrm{H}$ environment, causing bursts with durations of minutes. At the intermediate accretion regime $\left(10^{-9} \gtrsim \dot{M} /\left(M_{\odot} \mathrm{yr}^{-1}\right) \gtrsim 2 \times 10^{-10}\right)$ the helium ignites in an unstable fashion in a hydrogen-poor environment, causing bursts with durations smaller than $10 \mathrm{~s}$. At the lowest accretion regime $\left(\dot{M} \lesssim 2 \times 10^{-10} M_{\odot} \mathrm{yr}^{-1}\right)$ unstable hydrogen burning triggers a helium flash causing bursts with durations larger than $10 \mathrm{~s}$.

If the accretion rate is stable over long periods of time, periodic burst behavior is expected. It should always take the same amount of time to accrete enough matter to start the unstable burning again. This quasi-periodic burst behavior is observed in several burst sources, for example 4U 1820-30 (Haberl et al. 1987) or 4U 1705-44 (Langmeier et al. 1987), but only for limited periods of time. During other periods the occurrence of bursts appear completely a-periodic. An exception is GS 182624 whose bursts are always seen to recur quasi periodically (Ubertini et al. 1999; Cocchi et al. 2001b).

In this paper we describe the burst behavior of the nine most frequent X-ray bursters in the Galactic center region observed with the Wide Field Cameras. All are known X-ray bursters and most of them are persistently bright $\left(L_{\mathrm{X}} \sim 10^{36-37} \mathrm{erg} \mathrm{s}^{-1}\right)$. We compare these bursters with each other and with other bursters. The observations and the search for type I bursts are described in Sect. 2. In Sect. 3 we present the results. We start in Sect. 3.1 with the general properties of the nine burst sources. In Sect. 3.2 we discuss the wait time as a function of persistent emission for the bursters where this is possible. In Sect. 3.3 we compare the exponential decay times of the bursts with the theoretical regimes. Finally in Sect. 4.1 we start with a summary of the observations and compare our results with previous studies and in Sect. 4.2 discuss some implications for burst theory. We also derive some general properties of the population of X-ray bursters.

\section{Observations and data analysis}

The BeppoSAX satellite operated from May 1996 until May 2002 (Boella et al. 1997). During this period the Wide Field Cameras (Jager et al. 1997) onboard the satellite observed the Galactic center region each spring and fall with an average schedule of one day per week. This adds up to 12 Galactic center campaigns with a total net observation time of $7 \mathrm{Ms}$. The Wide Field Cameras (WFC) are two identical coded mask cameras with a $40^{\circ} \times 40^{\circ}$ field of view, a $5^{\prime}$ angular resolution, 2-28 keV bandpass and $20 \%$ spectral resolution (full width at half maximum at $6 \mathrm{keV}$ ). The large field of view combined with the good angular resolution makes it an excellent instrument to simultaneously observe a large fraction $(50 \%)$ of the low mass $\mathrm{X}$-ray binary (LMXB) population in our Galaxy when pointed at the Galactic center.

Each source in the field of view casts a shadow of the mask pattern on the detector. The detector accumulates the sum of differently shifted mask shadows. By cross-correlating this detector image with the mask pattern a sky image is reconstructed (e.g., in 't Zand 1992). This procedure is supplemented with a dedicated iterative cleaning algorithm (Hammersley et al. 1992). When no new sources are detected in the iterative process the background is estimated from the (supposedly) empty sky image. Lightcurves are constructed in the full bandpass for each detected source with a time resolution of $5 \mathrm{~s}$, which is a trade-off between the average duration of a type I burst ( $\simeq 10 \mathrm{~s}$ ) and the statistical quality of the data.

These lightcurves are searched for X-ray bursts in the following manner. We estimate the average flux and the standard deviation $(\sigma)$ for each orbit (about $60 \mathrm{~min}$ of net exposure time). If a bin is at least $4 \sigma$ above the average flux we mark this as a candidate burst. We estimate that we have, on average, only one false peak triggered as a burst per observation. A triggered bin is visually approved as a burst if the shape of the lightcurve around the bin can be described by a fast rise and exponential decay. If needed, this is done at $1 \mathrm{~s}$ time resolution. Thus, we detect all bursts with e-folding times $\gtrsim 2 \mathrm{~s}$ and peak fluxes in excess of $0.5 \mathrm{Crab}$. Bursts with lower peak intensities or shorter e-folding times do exist but this is only a minor fraction compared to the bursts detected (e.g. Cocchi et al. 2001b; van Paradijs et al. 1988b).

We also searched the lightcurve of all photons detected on the whole detector ("detector lightcurve") for X-ray bursts from sources whose persistent flux is below the detection limit of the WFC (see e.g., Cornelisse et al. 2002); note that this limit becomes worse toward the edge of the field of view. Detector lightcurves are created with a resolution of $1 \mathrm{~s}$. A running average of 50 bins is calculated and if at maximum 24 successive bins are $>4 \%$ above the average the first bin is labeled as a candidate burst. For all potential new bursts we cross-correlate the detector image again with the coded mask but only for the burst time interval. In this way a point source from which the burst originated may be identified. The sensitivity in this procedure is at most a factor of 2 worse than in the above mentioned procedure.

\section{Results}

\subsection{Global burst behavior}

A total of 1823 bursts have been detected from 37 sources in the Galactic center region, when not taking into account bursts from the Rapid Burster (MXB 1730-333) and the Bursting Pulsar (GRO J1744-29). For each burst we determined the exponential decay time in the total energy band $(2-28 \mathrm{keV})$, the peak flux and average persistent flux over the observation in WFC $\mathrm{cts} \mathrm{s}^{-1} \mathrm{~cm}^{-2}$. In Table 1 we give an overview of the nine most frequent bursters in the Galactic center region. All these sources are known X-ray bursters and have been studied in the past. The number of type I bursts detected with the WFC in other burst sources is too small for a meaningful statistical analysis.

In Fig. 1 we show RXTE All Sky Monitor (RXTE/ASM; Levine et al. 1996) lightcurves of the sources listed in Table 1. Most lightcurves show a smooth variation and no large fluctuations on a timescale of weeks. On the timescale of years a variation by a factor of roughly $50 \%$ is often present in these sources, and an apparent increase in burst rate (indicated by arrows) when the source becomes fainter. In contrast, the lightcurves of $4 \mathrm{U} 1705-440$ and $4 \mathrm{U} 1820-30$ show strong 

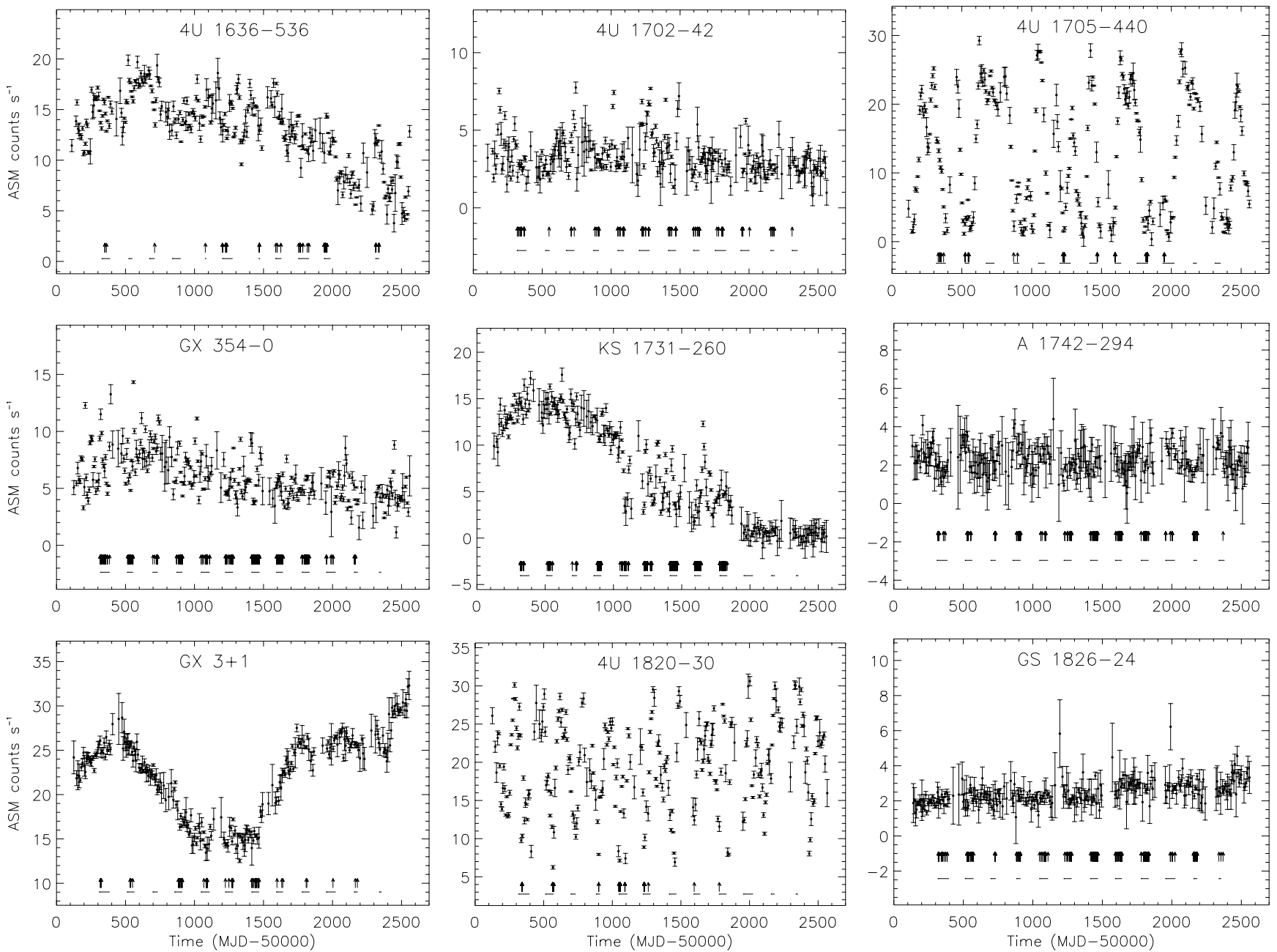

Fig. 1. ASM/RXTE lightcurves of 9 of the most frequent X-ray bursters in the WFC database. Each bin is a one week average. Below the lightcurve the WFC observations on these sources are indicated with horizontal bars. The arrows just above the horizontal bars indicate the times of type I bursts.

variations, and bursts are only observed when the flux is low. Note that bursts would have been easily detected at the highest observed persistent flux levels of all sources, because those levels are presumed to be still significantly below the Eddington limit.

To study the burst rate as a function of persistent flux we assigned to each burst the average WFC-measured flux over the complete observation in which the burst occurred. We also checked the average persistent flux in a 5-min time interval prior to each burst, but the difference with the aforementioned flux is within the errors. The persistent flux range was divided in 5 or 10 intervals of equal size. Only the flux range of KS 1731-260, the sole transient source in our sample, was divided in 10 bins with different bin sizes. For each flux interval we determined the total exposure time and the number of bursts; the latter with an error equal to the square root of this number (if no bursts are within an interval upper limits of $68 \%$ confidence were estimated). In Fig. 2 we show for each source the burst rate as a function of observed photon flux (bottom axis) and the derived energy flux (top axis). To get an indication of the conversion of photon flux to energy flux we generated for each source a spectrum for each campaign. We fitted the spectrum, assuming an absorbed thermal bremsstrahlung model, and we derived a mean conversion factor over all campaigns (corrected for absorption), see Table 1 . The spread in the conversion factors over all campaigns is about $10 \%$ for each source. In the WFC passband the assumption of a bremsstrahlung spectrum is good enough to derive fluxes; fluxes derived using other models give comparable results.

We notice in Fig. 2 that at the lowest flux levels KS 1731-260 shows an increase in burst rate with increasing persistent flux. When KS 1731-26 reaches $1.7 \times$ $10^{-9} \mathrm{erg} \mathrm{cm}^{-2} \mathrm{~s}^{-1}$, the burst rate drops by a factor of 5 and at higher flux levels the burst rate slowly decreases. 4U 1702-429, GX 354-0 and GS 1826-24 all show only an increasing burst rate, while $4 \mathrm{U} 1636-536$ is the only source that shows a decreasing burst rate. $4 \mathrm{U} 1705-44, \mathrm{GX} 3+1$ and $4 \mathrm{U} 1820-30$ show a drop by a factor of $\simeq 5$ in burst rate over a small range of persistent flux. A 1742-294 is the only source for which no clear trend is visible, and the burst rate stays constant over the total observed flux range. This source traces out the lowest fluxes within our sample. 
Table 1. Overview of the nine most frequent burster sources in the Galactic center region. They are ordered in decreasing number of bursts observed per source with the WFC. For each source a factor (conv.) in erg/count is derived to convert photon flux to energy flux in 2-28 keV. We also show the net exposure for each individual source and the distance as quoted in the literature. For each distance estimate we assume an error of $30 \%$. The distances are derived from: [1] Galloway et al. (2002), [2] Muno et al. (2000), [3] in 't Zand et al. (1999), [4] Lutovinov et al. (2001), [5] Muno et al. (2001), [6] Gottwald et al. (1989), [7] Augusteijn et al. (1998), [8] Kuulkers \& van der Klis (2000), [9] Heasley et al. (2000).

\begin{tabular}{lccccc}
\hline \hline Source & $\begin{array}{c}\text { \#bursts } \\
\text { WFC }\end{array}$ & $\begin{array}{c}\text { Exp. time } \\
(\mathrm{Ms})\end{array}$ & $\begin{array}{c}\text { Conv. } \\
\left(10^{-8}\right)\end{array}$ & $\begin{array}{c}d \\
(\mathrm{kpc})\end{array}$ & Ref. \\
\hline GX 354-0 & 423 & 7.4 & 1.9 & 5.4 & {$[1]$} \\
KS 1731-260 & 339 & 6.7 & 1.5 & 7.0 & {$[2]$} \\
GS 1826-24 & 260 & 6.5 & 1.9 & 8.0 & {$[3]$} \\
A 1742-294 & 178 & 7.0 & 2.2 & 8.5 & {$[4]$} \\
4U 1702-429 & 104 & 8.9 & 1.7 & 6.7 & {$[5]$} \\
4U 1705-44 & 66 & 8.7 & 1.8 & 8.9 & $\mathrm{a}$ \\
4U 1636-536 & 61 & 4.7 & 1.5 & 5.9 & {$[7]$} \\
GX 3+1 & 61 & 6.9 & 1.6 & 4.5 & {$[8]$} \\
4U 1820-30 & 49 & 7.1 & 1.6 & 7.6 & {$[9]$} \\
\hline
\end{tabular}

a: Distance estimated from [6].

\subsection{Wait times}

Several sources are known to show quasi-periodic burst recurrence times during certain periods. The best example is GS 1826-24, in 1996-1997 it exhibited a burst every $\simeq 6$ hours, and the burst wait times were constant within a few minutes for long periods of time (Ubertini et al. 1999; Cocchi et al. 2001b).

In the left panel of Fig. 3 we plotted the wait time as a function of the persistent flux for GS 1826-24. Most wait times appear to follow a straight line at the bottom of the figure. A second linear trend can clearly be distinguished above this line (and even two more above that). Given the fact that BeppoSAX has a 96-min low-earth orbit, it is probable that bursts are missed during data gaps and that multiples of the burst wait times are observed. We checked bursts with long wait times within one observation and find that for all of them the previous burst may have occurred during an earth occultation or South Atlantic Anomaly passage. From Fig. 3 we see that the wait time between the bursts decreases linearly with increasing persistent flux. We performed a least-squares fit on the bursts where the previous one is not missed to the function: $\Delta t=A F_{\text {pers }}+B$. The results for the parameters are given in Table 2. Assuming a wait time that is two times longer (i.e., doubling the numbers derived above) gives a good description of the trend followed by the bursts forming the second line from the bottom. This shows again that for these points the previous burst is missed. Note that formally the fit is not acceptable $\left(\chi_{v}^{2}=5.7,92\right.$ d.o.f.), but the general trend is clearly visible. This means that there are significant fluctuations in the wait time around the average relation.

From theory a linear relation between the burst rate (inverse of the wait time) and the persistent flux is expected, and no bursts are expected anymore when $F_{\text {pers }}=0 \mathrm{erg} \mathrm{cm}^{-2} \mathrm{~s}^{-1}$ (i.e. no accretion). We therefore tried to fit the relation: $\Delta t=$ $C / F_{\text {pers }}$. The result of the fit of parameter $\mathrm{C}$ is given in Table 2 .

Given the large number of bursts, we have also investigated the relation between the wait time and persistent flux for KS 1731-260 and GX 354-0. In the middle panel of Fig. 3 we show the results for KS 1731-260, and notice the strong suggestion of a linear dependency. However, this only applies to persistent flux levels below $0.14 \mathrm{WFC} c \mathrm{cts}^{-2} \mathrm{~s}^{-1}$. At higher persistent flux the wait time between bursts becomes apparently random. We fitted the relations as given above for the bursts with a persistent flux below 0.14 WFC cts cm $\mathrm{cm}^{-2} \mathrm{~s}^{-1}$ and where we expect that the previous burst is not missed. The best fit parameters are given in Table 2.

Also for GX 354-0 there vaguely appears to be a linear relation between the persistent flux and the wait time (right panel Fig. 3). However, the scatter is significantly larger than in the previous two cases, making a clear distinction between the different multiples of the wait time very difficult. Therefore, an iterative process was used to search for bursts where we expect that the previous one is not missed. We simultaneously fitted a straight line (the single wait time line) plus several lines at multiples of the wait time. The bursts closest to the single wait time line were attributed to this line and used for a least-square fit to get a better estimate. This process was continued until a best fit was found. The bursts attributed to the single wait time line were also used to fit the relation: $\Delta t=C / F_{\text {pers }}$. The results are summarized in Table 2.

For the other six sources the number of subsequent bursts with a wait time of less than one day becomes very small, and the data does not allow the verification of a linear relation.

We converted the fit parameters as given in Table 2 from the observed flux to luminosities using the conversion factors and distances as given in Table 1 . For the parameter $C$ the values are $(2.5 \pm 1.5) \times 10^{36},(0.8 \pm 0.5) \times 10^{36}$ and $(1.9 \pm 1.1) \times 10^{36} \mathrm{erg} \mathrm{s}^{-1}$ for GS 1826-24, KS 1731-260 and GX 354-0, respectively (taking into account an error of $30 \%$ in the distance). Although the errors are very large, these slopes are the same within their errors. Therefore, the burst rate may be a unique function of the persistent flux.

\subsection{Decay times}

Another important burst parameter is the e-folding decay time. As discussed in Sect. 1, this diagnoses the composition of the burst fuel. To derive the decay time for each burst we generated lightcurves with a $1 \mathrm{~s}$ time resolution. A running average of $5 \mathrm{~s}$ was used to determine the moment of the peak flux. The persistent emission level and the decay time are then simultaneously fitted with a constant and exponential, respectively. We took the bin in which the peak flux was reached as the first data point. In Fig. 4 we show the decay times as a function of persistent emission for the nine sources. We here discuss GX 354-0, KS 1731-260 and GS 1826-24 in more detail and compare them with the other sources.

GX 354-0 only shows bursts with decay times shorter than $\simeq 10 \mathrm{~s}$ at all flux levels. The same applies to $4 \mathrm{U} 1636-536$, 


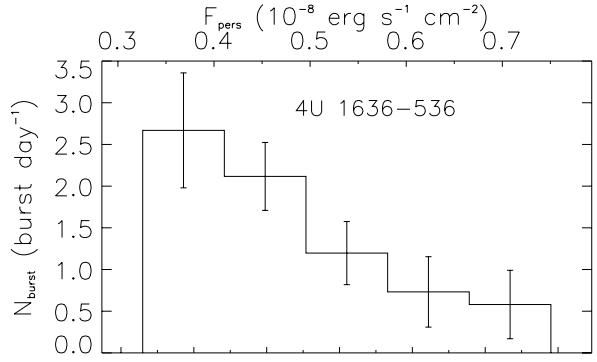

$\begin{array}{lllllll}0.20 & 0.25 & 0.30 & 0.35 & 0.40 & 0.45 & 0.50\end{array}$
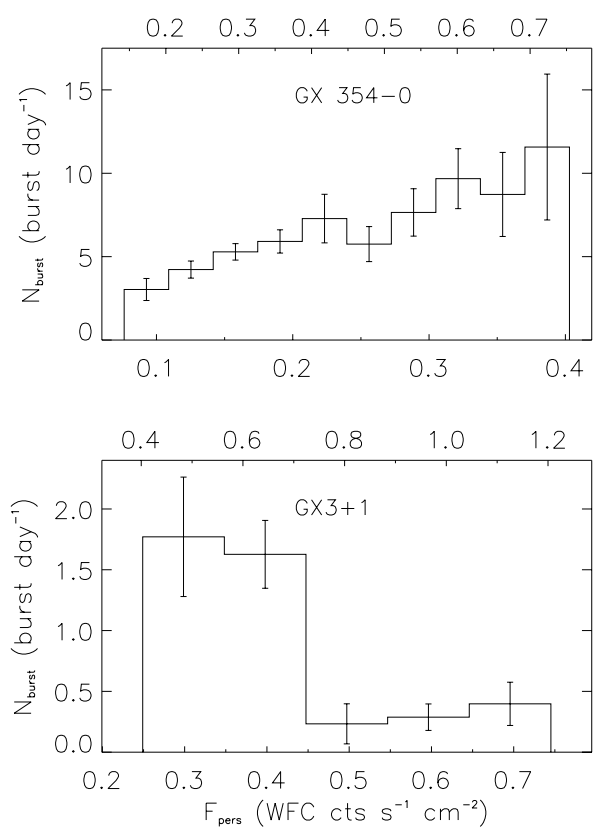
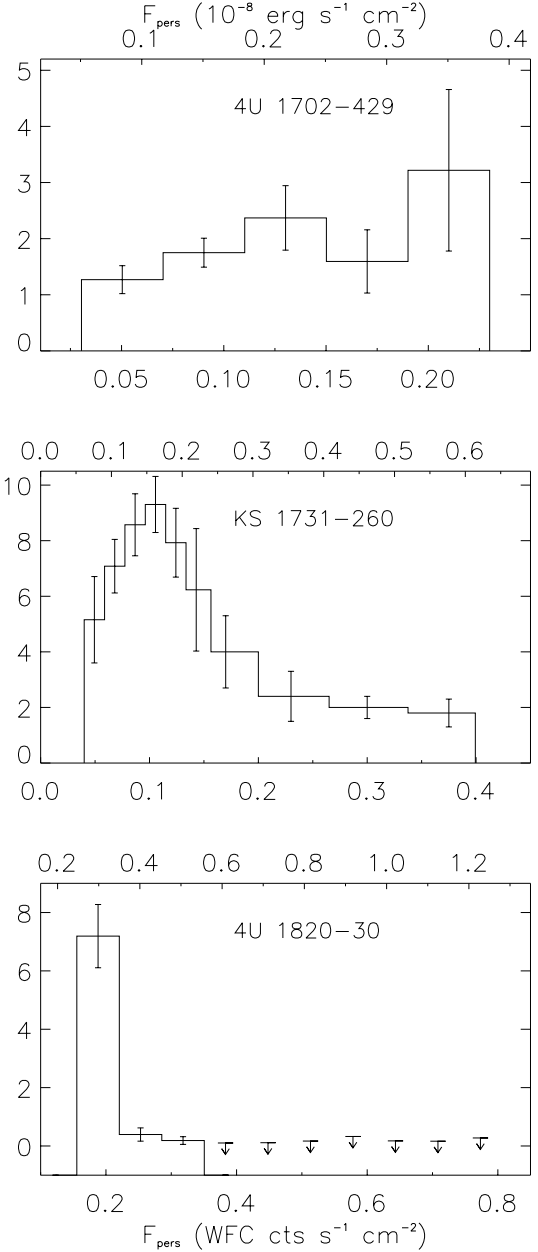

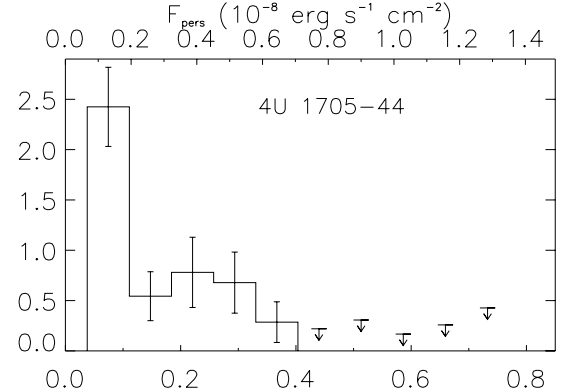

$\begin{array}{llllllll}0.04 & 0.06 & 0.08 & 0.10 & 0.12 & 0.14 & 0.16\end{array}$
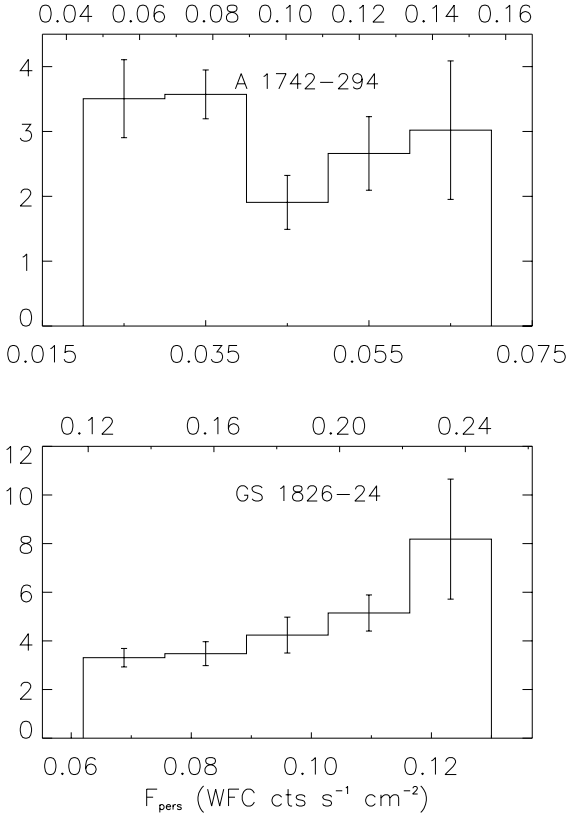

Fig. 2. Burst rate, $N_{\text {burst }}$, as a function of the observed count rate, $F_{\text {pers }}$ (WFC cts s${ }^{-1} \mathrm{~cm}^{-2}$; bottom axis) and the persistent flux, $F_{\text {pers }}$ $\left(10^{-8} \mathrm{erg} \mathrm{s}^{-1} \mathrm{~cm}^{-2}\right.$; top axis) for the nine frequent X-ray bursters in the galactic center region. The photon and energy flux are for a bandpass of $2-28 \mathrm{keV}$.

GX $3+1$ and 4U 1820-30. GS 1826-24 shows a large range of decay times at all persistent flux levels, but almost no bursts below $10 \mathrm{~s}$ are observed (i.e., less than $5 \%$ of all bursts). Of the nine sources, this is the only one that shows this behavior.

Two trends can be observed for the decay times of KS 1731-260. At high persistent flux ( $\left.\gtrsim 0.14 \mathrm{WFC} \mathrm{cts} \mathrm{s} \mathrm{cm}^{-2}\right)$ all decay times are below $10 \mathrm{~s}$, as for e.g. GX 354-0. At lower fluxes the spread in decay times increases rapidly and most bursts have decay times well above $10 \mathrm{~s}$, as for GS 1826-24. However, in contrast to GS 1826-24, still a significant fraction of bursts show decay times below $10 \mathrm{~s}$ (about 30\%). The same behavior is also suggested by the figures for $4 \mathrm{U} 1702$ 429, 4U 1705-44 and A 1742-294.

A spectral change instead of a change in persistent emission could be the only indication of a change in mass accretion (van der Klis et al. 1990). This could explain the occurrence of both long and short bursts at low persistent flux. We therefore investigated the low persistent flux levels of KS 1731-260 in a little more detail. At MJD 51799.60 and MJD 51799.72 there were bursts with decay times of $4.7 \pm 0.1 \mathrm{~s}$ and $20.8 \pm 3.5 \mathrm{~s}$, respectively. Due to the low flux level of the source full resolution spectra do not have enough statistics, and we resorted to the study of hardness ratios. The WFC passband was divided in two channels from $2-6 \mathrm{keV}$ and $6-28 \mathrm{keV}$, and derived hardness ratios by dividing the count rates in the $6-28 \mathrm{keV}$ with the count rates in the $2-6 \mathrm{keV}$ band. We found hardness ratios of $0.68 \pm 0.07$ and $0.70 \pm 0.08$ for the periods prior to the two bursts, respectively. We note that the average persistent flux stayed constant at 0.111 and $0.115 \mathrm{WFC} \mathrm{cts} \mathrm{s}^{-1} \mathrm{~cm}^{-2}$ in these periods. The $1 \sigma$ statistical fluctuations at one minute time resolution are $24 \%$ and $30 \%$, respectively. We conclude that no significant changes occurred between the two bursts.

The transition from short bursts to long/short bursts in $\mathrm{KS} \mathrm{1731-260} \mathrm{is} \mathrm{rapid.} \mathrm{Therefore,} \mathrm{an} \mathrm{observation}$ (at MJD 51637) where the persistent emission is at this transition was analyzed in more detail. A spectrum was derived for this observation, assuming an absorbed bremsstrahlung spectrum with a hydrogen absorption column of $1.3 \times 10^{22}$ atoms cm ${ }^{-2}$ (Predehl \& Schmitt 1995). A temperature of $21.4 \pm 3.4 \mathrm{keV}$ and an unabsorbed flux of $(2.4 \pm 0.3) \times 10^{-9} \mathrm{erg} \mathrm{cm}^{-2} \mathrm{~s}^{-1}(2-28 \mathrm{keV})$ was estimated A power law spectrum with a photon index of $1.64 \pm 0.06$ gives a flux of $(2.6 \pm 0.2) \times 10^{-9} \mathrm{erg} \mathrm{cm}^{-2} \mathrm{~s}^{-1}(2-28 \mathrm{keV}$; corrected for absorption). Converting these numbers to a mass accretion rate, assuming standard neutron star parameters ( $R=10 \mathrm{~km}, M=1.4 M_{\odot}$, solar abundances) and $100 \%$ 
GS $1826-24$

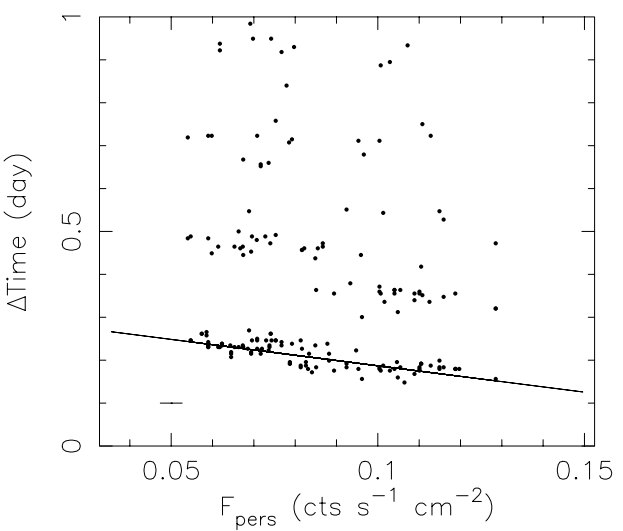

KS $1731-260$

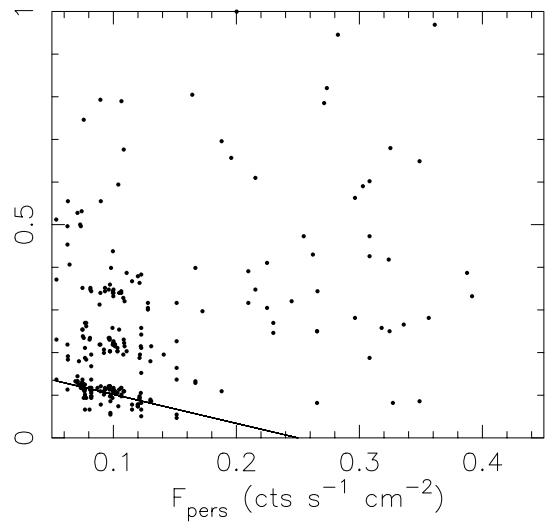

GX 354-0

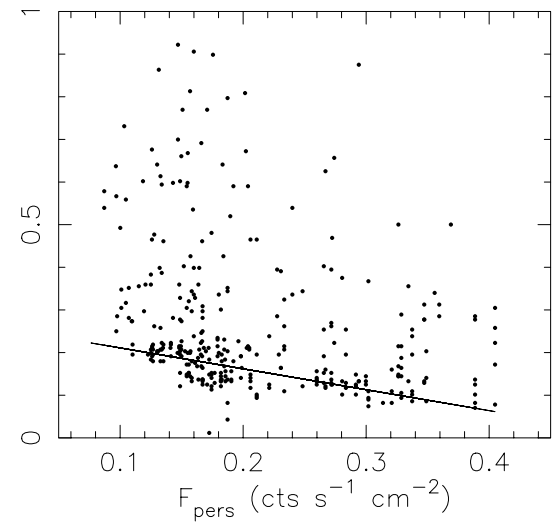

Fig. 3. The wait time, $\Delta$ Time, as a function of persistent flux, $F_{\text {pers }}$, for the sources GS 1826-24 (left panel) and KS $1731-260$ (middle panel) and GX 354-0 (right panel). A typical error in the flux for GS 1826-24 is indicated at the bottom left, while the error in the flux for the other two sources is as large as a dot. The error in the wait time for all sources is much smaller than the size of a dot. A best linear fit is drawn for the bursts for which the previous burst is not missed.

Table 2. Best fit parameters for a relation where the wait time is proportional $(A$ and $B)$ or inversely proportional $(C)$ to the persistent flux. Due to the large $\chi_{v}^{2}$ the formal errors on the parameters have no meaning.

\begin{tabular}{lccc}
\hline \hline parameter & GS 1826-24 & KS 1731-260 & GX 354-0 \\
\hline$A$ & $-1.23 \pm 0.10$ & $-0.68 \pm 0.09$ & $-0.49 \pm 0.07$ \\
$B$ & $0.31 \pm 0.01$ & $0.17 \pm 0.01$ & $0.26 \pm 0.01$ \\
$\chi_{v}^{2}$ (d.o.f.) & $5.7(92)$ & $20(100)$ & $266(202)$ \\
\hline$C$ & $0.017 \pm 0.001$ & $0.009 \pm 0.001$ & $0.029 \pm 0.001$ \\
$\chi_{v}^{2}$ (d.o.f.) & $6.2(93)$ & $38(101)$ & $234(203)$ \\
\hline
\end{tabular}

efficiency in converting gravitational energy to radiation, gives $1.3 \times 10^{-9} M_{\odot} \mathrm{yr}^{-1}$. This is similar to the estimated mass accretion rate of GX $3+1$ at its transition (den Hartog et al. 2002).

\section{Discussion}

\subsection{Observational summary}

Our observations of the burst rate as a function of luminosity for the nine frequent X-ray bursters are summarized in Fig. 5. In order to compare sources we calculated the $2-28 \mathrm{keV} \mathrm{lu}-$ minosity from the flux and the distances as listed in Table 1. Given that the distance has an estimated accuracy of $30 \%$ and that the $2-28 \mathrm{keV}$ luminosity is only a crude indicator of the accretion rate, we estimate that the luminosity correspondence between sources is accurate to about a factor of 2 . The following conclusion can be drawn from Fig. 5 (and 2): the burst rate shows trends with luminosity that are consistent over all sources. Between 1 and $2 \times 10^{37} \mathrm{erg} \mathrm{s}^{-1}$ the burst rate peaks. Above that the burst rate drops fast by roughly a factor of 5 (first observed in GX 3+1 by den Hartog et al. 2002, and now also seen for 4U 1820-30, KS 1731-260, and 4U 170544). Below that there is a smooth increase towards the peak.
Above $\simeq 5.5 \times 10^{37} \mathrm{erg} \mathrm{s}^{-1}$ no bursts are seen anymore. The latter is confirmed by observations of brighter low-mass X-ray binaries in the same field with presumably similar distances such as GX 9+1, GX 349+2, GX 340+0, GX 17+2, GX 13+1 and GX 5-1. None of these were seen to burst by the WFC. The general trends in burst rate were known previously. The knowledge that our work adds is 1) that there does seem to be a rather consistent burst rate behavior from one burster to another, and 2) that there is a rather discrete transition in this behavior between 1.4 to $2.1 \times 10^{37} \mathrm{erg} \mathrm{s}^{-1}$.

Our searches for (quasi-)periodicity in burst recurrence were meaningful in three sources: GX 354-0, KS 1731-260 and GS 1826-24. The presence of quasi-periodicities is most obvious in GS 1826-24 (see also Ubertini et al. 1999 and Cocchi et al. 2001b), present in KS 1731-260, but only suggestive in GX 354-0. The quasi-periodicity is only present during times when the persistent flux is below that for the peak burst rate, as is most clearly demonstrated by KS 1731-260 which, thanks to its transient nature, traces a relatively wide range of fluxes. GS 1826-24 never leaves this domain which explains why its bursts always recur quasi-periodically. The same appears to apply to GX 354-0. Quasi-periodicity has been seen previously with EXOSAT in a number of other sources: EXO 0748-676 (Gottwald et al. 1986), 4U 1705-44 (Gottwald et al. 1989), Ser X-1 (Sztajno et al. 1983) and 4U 1636-536 (Lewin et al. 1987) but only for a limited amount of time. Our observations for the first time show empirically that the quasi-periodicity is restricted to a very particular luminosity range and that there is a narrow positive relationship between burst frequency and the persistent flux.

Our determinations of burst decay times in KS 1731-260 and 4U 1705-44 suggest a clear correspondence between decay time, burst rate behavior and quasi-periodicity, in the sense that there is a clear transition at a luminosity between 1.4 and $2.1 \times$ $10^{37} \mathrm{erg} \mathrm{s}^{-1}$. However, the decay times observed in GX $3+1$ and $4 \mathrm{U} 1705-44$ do not follow this trend despite tracing out similar ranges in luminosity (formally the same applies to $4 \mathrm{U} 1820-30$ 

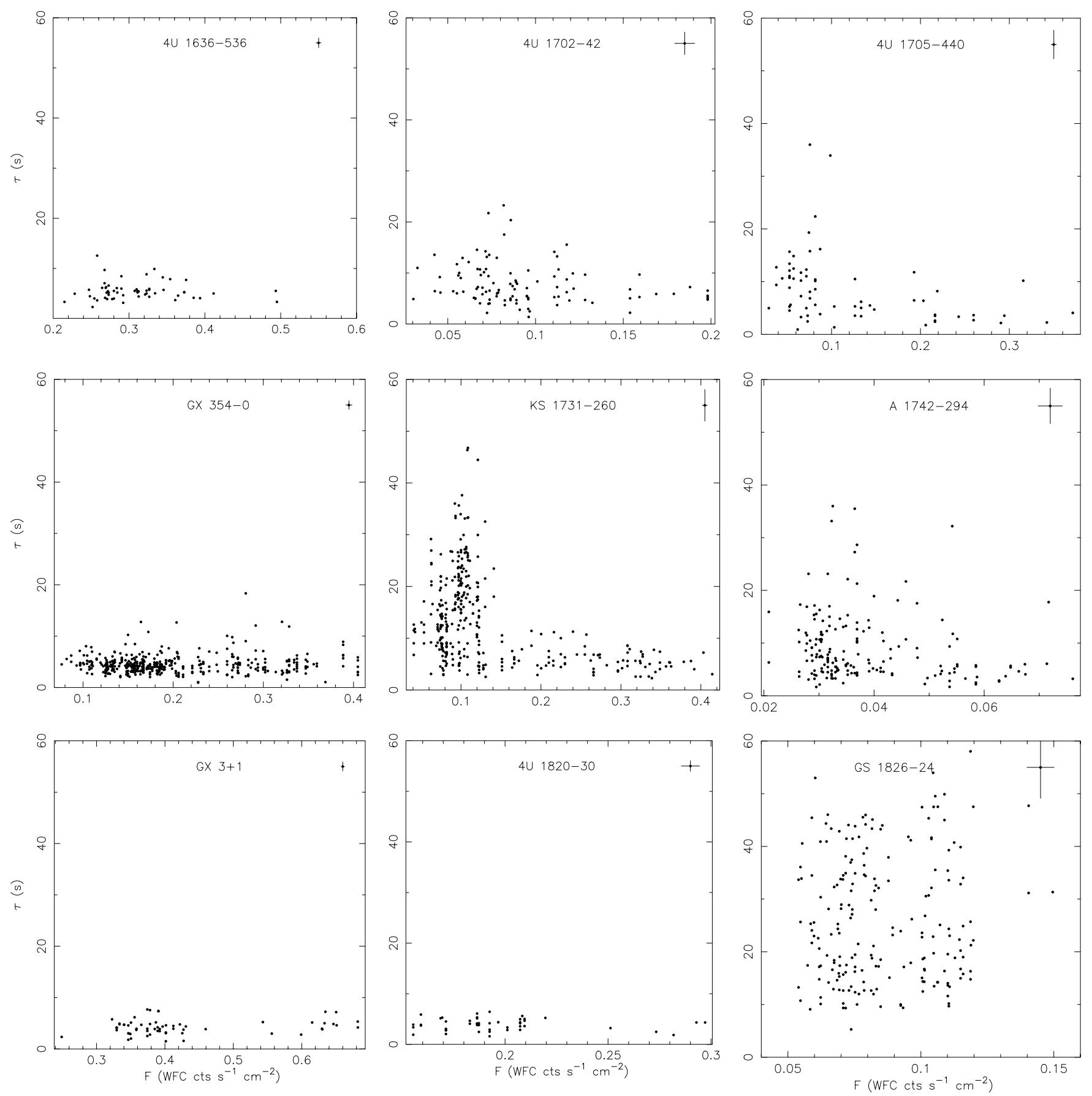

Fig. 4. The exponential decay time, $\tau$, as a function of persistent flux, $F$, for the nine most frequent burst sources observed with WFC. In the upper-right corner of each panel a typical error-bar is shown.

but here we know that the decay times cannot be long because the mass donor is proven to be a helium white dwarf; Stella et al. 1987).

To summarize: the central finding in our study is the likely identification of a single luminosity between 1.4 to $2.1 \times$ $10^{37} \mathrm{erg} \mathrm{s}^{-1}$, consistent with a single mass accretion rate, where the bursting behavior changes in three basic ways: going to higher luminosities, bursts 1) become rather quickly a factor of 5 less frequent, 2) stop recurring quasi-periodically, 3) stop being long.

\subsection{Theoretical interpretation}

From theory it is expected that long bursts can only be due to helium flashes in a hydrogen-rich environment, which is predicted to occur at the highest or lowest accretion regimes (Fujimoto et al. 1981; Bildsten 1998; see also introduction). Given the fact that in our sample only short bursts are observed at higher luminosities, we may identify the transition in burst behavior with the transition from the lowest to the middle accretion regime. 


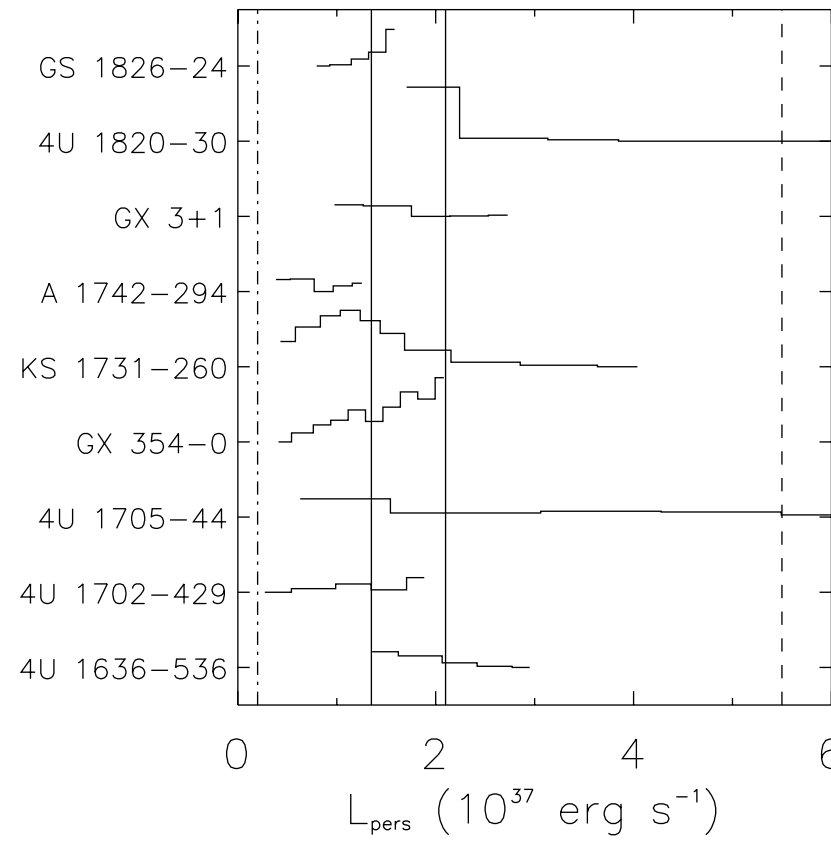

Fig. 5. A schematic diagram of the burst rate as a function of luminosity for the nine X-ray burst sources (see Fig. 2). Between the solid lines the region is indicated where the burst rate drops by a factor of 5 . No type I bursts were observed at luminosities above the dashed line. The dotted-dashed line indicates the theoretical predicted transition from hydrogen-rich bursts to pure helium bursts.

At the lowest luminosities the helium flash is triggered by unstable hydrogen burning (Fujimoto et al. 1981). Between two bursts no hydrogen is burned, and it is only the accretion of matter that increases the pressure and temperature to high enough values to start this burning. If we assume that the accretion flow is stable then the burst wait time is only dependent on the accretion rate, and a quasi-periodic behavior is not unexpected, as is a narrow relationship between its frequency and the persistent flux.

In the middle accretion regime, bursts take place in a pure helium shell which is fed by stable hydrogen burning in a layer above that. When a critical temperature and pressure are reached the helium ignites. Here the onset of the bursts is determined by the heating of the shell due to the hydrogen burning and the accretion. But more importantly, the onset of helium burning is very sensitive to the temperature (Bildsten 1998), making it highly dependent on local perturbations in the hydrogen burning. This means that local conditions determine the onset (and thus the wait time) of a burst, and quasi-periodic behavior is not readily expected anymore.

Hydrogen starts burning in an unstable fashion at lower column depths than helium (Joss 1977). This means that the conditions for triggering an X-ray burst in the lowest accretion regime are reached after accreting a smaller amount of matter than in the middle accretion regime, and a higher burst rate is expected. Assuming that only the accretion rate can set the condition for the start of unstable hydrogen burning, it is expected that this transition happens in a fairly small range of accretion rates. We remark that recently an interesting phenomenon was detected of quasi periodic oscillations (QPOs) at $7-8 \mathrm{mHz}$ in a few bursting LMXBs which appear to coincide with this transition and whose presence is coupled to the occurrence of type I bursts (Revnivtsev et al. 2001). Revnivtsev et al. speculate that these QPOs are related to special modes of nuclear burning. Support for this hypothesis is provided from trends in $\mathrm{kHz}$ QPOs observed in one of those sources (Yu \& van der Klis 2002).

We have for the first time shown that the largest decrease in burst rate towards higher fluxes is coincident with the onset of stable hydrogen burning. Thereby, the above-mentioned explanation for the apparently sudden decrease in burst rate may partly resolve a long-standing problem for explaining decreasing burst rates. Van Paradijs et al. (1988) explained this by invoking increased stable helium burning with increasing accretion rate. However, such burning is not expected to occur at sub-Eddington accretion rates (Fujimoto et al. 1981). Bildsten (2000) suggested that non-global accretion on the neutron star (Marshall 1982; Inogamov \& Sunyaev 1999) may explain the decreasing burst rate: the accretion area may be smaller than the neutron star surface so that the local mass accretion rate is higher than the globally measured one.

It is unclear how the burst rate behaves above the transition. Direct measurements (Fig. 2) are ambiguous: it may be constant or slowly decreasing. The rarity of X-ray bursts for more luminous sources suggests that there is a decrease, but observations in this domain are subject to strong selection effects as was already noted by other investigators (i.e., since the persistent emission is already close to Eddington there is hardly any room for flux increases by bursts). If the decrease is real, that does need to be explained, perhaps in a manner as proposed by Van Paradijs et al. (1988) and Bildsten (2000).

So far, we have dealt with observations that are explainable in current burst theory. There are three observational facts for which this is more difficult. The first is that short bursts seem to be rather common in the low accretion regime. Long bursts would be expected because the flashes occur in a hydrogen-rich layer. This problem was recently also recognized by den Hartog et al. (2002) for the specific case of GX 3+1. Also, short type I bursts have been detected with extremely low persistent fluxes (e.g., a short, 2.6 s, burst from SAX J2224.9+5421 was quickly and deeply followed up in X-rays and no persistent source was found with a 2-10 keV upper limit of $1.3 \times 10^{-13} \mathrm{erg} \mathrm{cm}^{-2} \mathrm{~s}^{-1}$; see Cornelisse et al. 2002). Fujimoto et al. (1981) briefly sketch an alternative path to trigger bursts in the lowest regime that could explain this. If the unstable hydrogen burning in the bottom shell does not trigger the helium burning instantaneously it will cause temporary stable hydrogen burning in the higher shells. The unstable hydrogen burning is not observable (Joss 1977). A pure helium layer will be built up by the stable hydrogen burning and it will probably take a number of invisible hydrogen flashes to trigger a helium flash in this layer, very much like in the middle accretion regime, with a short burst as a result.

It is unclear what determines the varying mix of short and long bursts (100\% short bursts for GX 3+1 and GX 354-0, less than 5\% for GS 1826-24, and in-between percentages for other sources). We suspect that variability of the persistent flux may be an issue. If we compare the ASM lightcurves in Fig. 1 of 
KS 1731-260 and GS 1826-24 we notice that KS 1731-260 is more variable at comparable luminosities (between MJD 51000 and MJD 51700). During the last WFC campaign (around MJD 51800) only long bursts are observed for KS 1731-260 and the variability becomes comparable to GS 1826-24. This would indicate that low variability, (i.e., a smooth accretion rate) gives rise to solely long X-ray bursts, while a more inhomogeneous accretion rate gives rise to incidental pure helium flashes, and this would lead to the following naive picture. An inhomogeneous accretion flow would give rise to large variation in the local accretion rate. These variations could at certain times be high enough to start stable (or unstable) hydrogen burning, and the hydrogen would be depleted in the burning layers. This would give rise to hydrogen poor bursts. For a homogeneous accretion flow, where the local accretion rate stays constant over time, hydrogen burning would not start and long burst would be observed.

Another issue unexplainable by current burst theory is the different peak burst rate between different sources. A bi-modal distribution appears to be present. GX 354-0, KS 1731-260, 4U 1820-30 and GS 1826-24 all have a peak burst rate of about 9 bursts day $^{-1}$, while the other sources have a peak burst rate of about 2.5 bursts day $^{-1}$. EXOSAT observations of 4U 1636536 showed a burst rate of 8 bursts day $^{-1}$, more in line with the first group (Lewin et al. 1987). Given the large uncertainty in the distance it could well be possible that during the WFC observations the luminosity of $4 \mathrm{U} 1636-536$ is not low enough for such high burst rates, and this might be the case for the other sources. We have no good explanation for this apparent bi-modality.

As was already noted by van Paradijs (1988), there exists a large discrepancy between theory and observations with regards to the mass accretion rate at which the transition takes place from the lowest accretion regime to the middle regime. We find a value of (1.4-2.1) $\times 10^{-9} M_{\odot} \mathrm{yr}^{-1}$, thus confirming the values found by van Paradijs et al. (1988), while theory predicts 1 to $2 \times 10^{-10} M_{\odot} \mathrm{yr}^{-1}$ (Fujimoto et al. 1981; Bildsten 1998; indicated in Fig. 5 with a dash-dotted line). The theoretical values do depend somewhat on assumptions for a number of neutron star parameters (radius, mass, etc.) and conditions in the neutron star (abundances of CNO elements, opacity, etc.) and become somewhat uncertain due to uncertainties in the distance. This results in substantial margins in the theoretical value from source to source of a factor of three. Still, this is insufficient to explain the discrepancy.

Bildsten (2000) suggests that the discrepancy can be resolved if the transition is not from the lower to the middle regime but from the upper to the middle regime. This may be accomplished if the area over which the accretion takes place is smaller than the neutron star and grows faster than linearly with the global accretion rate. Thus, the mass accretion rate per unit area diminishes as the global accretion rates grows and the source moves from the middle to the lower regime with increasing persistent fluxes. This idea is based on a suggestive radius versus persistent flux trend observed in EXO 0748-676 (Gottwald et al. 1986). It remains to be seen from a complete analysis of our sample, involving time-resolved spectroscopy of all bursts, whether the radius shows such trends with persistent flux. However, a partial result by den Hartog et al. (2003) on GX 3+1 shows no indications for such a trend. Furthermore, the measurements of one source in particular strongly suggest that this effect is not important: KS 1731-260 is the only frequent bursters that exhibits a large dynamic range in persistent flux due to it being transient. The source gradually dies out in our observations and traverses from high global mass accretion rates to a negligible ones. Our observations show that once it exited the middle regime, it never changed regime again. It seems unlikely that KS 1731-260 remains in the upper mass accretion rates per unit area regime all the way down to negligible global accretion rates. Therefore, our data suggest that the explanation as put forward by Bildsten (2000) for the discrepancy in predicted and observed threshold value between lower and middle accretion burst regime, cannot be general. This is further supported by the quasi-periodicity that we find in the burst recurrence for KS 1731-260 in this regime as well as GS 1826-24. Such a form of stability is more likely to happen if the trigger criterion for the flash is dependent on fewer parameters. In the lower burst regime, where the hydrogen is first ignited, the trigger is only dependent on pressure while in the upper regime, where the helium first ignites, it also depends on the local temperature.

\section{Summary}

Thanks to the wide (50\%) and long (7 Ms) coverage of the population of low-mass X-ray binaries we detected an unprecedented large number of type I X-ray bursts for nine sources which enabled us to perform a comparative study of bursting behavior which was hitherto not possible. We were able to accurately detect systematic trends in burst rate, burst duration and burst recurrence periodicity and to put them under the common denominator provided by current burst theory. Our central finding is that most of the trends in bursting behavior are driven by the onset of stable hydrogen burning in the neutron star atmosphere. Furthermore, we notice three new observational facts which are more difficult to explain with current burst theory: the presence of short pure-helium bursts at the lowest accretion regimes, the bimodal distribution of peak burst rates, and an accretion rate at which the onset of stable hydrogen burning occurs that is ten times higher than predicted. Finally, we note that our investigation is the first to signal quasiperiodic burst recurrence in KS 1731-260, and a clear proportionality between the frequency of the quasi-periodicity and the persistent flux in GS 1826-24 and KS 1731-260.

\section{References}

Augusteijn, T. J., van der Hooft, F., de Jong, J. A., van Kerkwijk, M. H., \& van Paradijs, J. 1998, A\&A 332, 561

Bildsten, L. 1998, in The many faces of neutron stars, ed. J. Bucheri, J. van Paradijs, \& M. A. Alpar (Dordrecht: Kluwer), 419

Bildsten, L. 2000, in Cosmic explosions, AIP, ed. S. Holt, \& W. Zhang, E65

Boella, G., Butler, R., Perola, G., et al. 1997, A\&AS, 122, 299

Cocchi, M., Bazzano, A., Natalucci, L., et al. 2001a, A\&A, 378, L37 
Cocchi, M., Bazzano, A., Natalucci, L., et al. 2001b, in Adv. Space Res., 28, 275 (Elsevier)

Cornelisse, R., Verbunt, F., in 't Zand, J. J. M., et al. 2002, A\&A, 392, 885

den Hartog, P. R., in 't Zand, J. J. M., Kuulkers, E., et al. 2003, A\&A, 400, 633

Fujimoto, M. Y., Hanawa, T., \& Miyaji, S. 1981, ApJ, 247, 267

Galloway, D. K., Psaltis, D., Chakrabarty, D., \& Muno, M. P. 2002, ApJ, submitted [astro-ph/0208464]

Gotthelf, E., \& Kulkarni, S. 1997, ApJ, 490, L161

Gottwald, M., Haberl, F., Parmar, A. N., \& White, N. E. 1986, ApJ, 308,213

Gottwald, M., Haberl, F., \& Langmeier, A. 1989, ApJ, 339, 1044

Grindlay, J., \& Heise, J. 1975, IAUC 2879

Haberl, F., Stella, L., White, N. E., Gottwald, M., \& Priedhorsky, W. C. 1987, ApJ, 314, 266

Hammersley, A., Ponman, T., \& Skinner, G. K. 1992, Nuc. Instr. Meth. Phys. Res. A311, 585

Heasley, J. N., Janes, K. A., Zinn, R., et al. 2000, AJ, 120, 879

Inogamov, N. A., \& Sunyaev, R. A. 1999, AstL, 25, 269

Jager, R., Mels, W., Brinkman, A., et al. 1997, A\&AS, 125, 557

Joss, P. C. 1977, Nature, 270, 211

van der Klis, M., Hasinger, G., Damen, E., et al. 1990, ApJ, 360, L19

Kuulkers, E., \& van der Klis, M. 2000, A\&A, 356, L45

Kuulkers, E., Homan, J., van der Klis, M., Lewin, W. H. G., \& Mendez, M. 2002, A\&A, 382, 947

Langmeier, A., Sztajno, M., Hasinger, G., \& Trümper, J. 1987, ApJ, 323, 288

Levine, A. M., Bradt, H., Cui, W., et al. 1996, ApJ, 469, L33
Lewin, W. H. G., Penninx, W., van Paradijs, J., et al. 1987, ApJ, 319, 893

Lewin, W. H. G., van Paradijs, J., \& Taam, R. 1993, Space Sci. Rev. 62,223

Lutovinov, A. A., Grebenev, S. A., Pavlinsky, M. N., \& Sunyaev, R. A. 2001, AstL, 27, 501

Marshall, H. L. 1982, ApJ, 260, 815

Muno, M. P., Fox, D. W., Morgan, E. H., \& Bildsten, L. 2000, ApJ, 542,1016

Muno, M. P., Chakrabarty, D., Galloway, D. K., \& Savov, P. 2001, ApJ, 553, L157

van Paradijs, J., Penninx, W., \& Lewin, W. H. G. 1988a, MNRAS, 233, 437

van Paradijs, J., Penninx, W., Lewin, W. H. G, Sztajno, M., \& Truemper, J. 1988b, A\&A, 192, 147

Predehl, P., \& Schmitt, J. H. M. M. 1995, A\&A, 293, 889

Revnivtsev, M., Churazov, E., Gilfanov, M., \& Sunyaev, R. 2001, A\&A, 372, 138

Stella, L., White, N. E., \& Priedhorsky, W. 1987, ApJ, 312, L17

Sztajno, M., Basinska, E. M., Cominsky, L. R., Marshall, F. J., \& Lewin, W. H. G. 1983, ApJ, 267, 713

Ubertini, P., Bazzano, A., Cocchi, M., et al. 1999, ApJ, 514, L27

Yu, W., \& van der Klis, M. 2002, ApJ, 567, L67

in 't Zand, J. J. M. 1992, A coded-mask imager as monitor of galactic X-ray sources, Ph.D. Thesis, Utrecht University

in 't Zand, J. J. M., Heise, J., Kuulkers, E., et al. 1999, A\&A, 347, 89

in 't Zand, J. J. M. 2001, in Exploring the gamma-ray universe, ed. A. Gimenez, V. Reglero, \& C. Winkler (ESA Pub. Div.), 463 